\title{
Whither COVID-19 vaccines?
}

\author{
Nature Biotechnology convenes a group of experts to provide their insights into SARS-CoV-2 vaccines in development.
}

Galit Alter is at Harvard Medical School and the Ragon Institute, Boston, MA, USA. Kate Bingham is at SV Health Managers, London, UK, and chairs the UK Vaccines Taskforce.

Larry Corey is at the Fred Hutchinson Cancer Research Center, Seattle, WA, USA. James Dahlman is at Georgia Institute of Technology and Emory University, Atlanta, GA, USA.

Nick Jackson is at Coalition for Epidemic Preparedness Innovations, Shanghai, China. John Moore is at Weill Cornell Medical School, New York, NY, USA.

Rino Rappuoli is at GlaxoSmithKline, Siena, Italy.

Questions swirl around trailblazing COVID-19 vaccines as they enter late-stage clinical testing (Tables 1 and 2), with anticipation building for an Emergency Use Authorization in the United States. How is broadening knowledge of immune responses to SARS-CoV-2 informing vaccine research? What are the pros and cons of the different vaccine modalities in discovery and clinical development? What are the key challenges associated with clinical testing, and what are their implications for safety and efficacy of immunization programs in the broader global population? And what issues related to regulatory oversight, manufacturing and distribution are likely to be important in the rollout of a COVID-19 vaccine?

How has research on the cellular and humoral immunity to SARS-CoV-2 and other coronaviruses informed vaccine development?

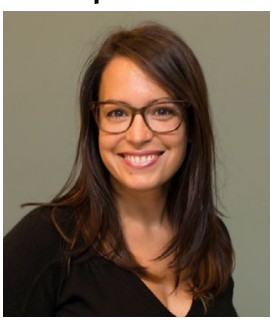

Galit Alter, Harvard Medical School and Ragon Institute.

\section{Galit Alter:}

Correlates of protection do not need to mimic correlates of resolution of disease. Immune mechanisms that govern clearance of an existing infection need not even include antibodies. However, one can learn about the specific footprints of the most potent neutralizing antibodies, about viral evolution/evasion, about additional 'backup' immune mechanisms that may be critical for eradication of an infection, and about the role of additional intrinsic immune phenomena that may govern asymptomatic infection or long-lived shedding from viral pathogenesis studies.
Vaccine studies show that neutralizing antibodies alone do seem to be protective against SARS-CoV-2 infection, although emerging data point to additional antibody effector functions and cellular immune responses that may support the complete elimination of the virus.

Kate Bingham: No vaccine has ever been developed against any human coronavirus, but there are good coronavirus vaccines for chickens and pigs. Although it is clear that both SARS-CoV-1 and SARS-CoV-2 trigger a strong immune response in most people, several studies have shown that antibody titers in those recovering from the virus decline within a few months of infection. This observation may not be surprising - if a person has successfully fought off the infection, you would expect their antibodies to drop to a lower, baseline level. So it is essential to run phase 3 safety and efficacy studies of vaccines to gain a deeper understanding of the immune responses generated to assess which vaccines confer protection against COVID-19, both to avoid infection (namely, via induction of strong mucosal immunity) and to prevent shedding. It is likely that memory $\mathrm{T}$ cells and $B$ cells contribute to long-term immunity and protection. Happily, we are in an era of unprecedented capacity to assess the immune response.

The UK government's Vaccine Taskforce [VTF] is investing in building standardized, accredited assays, including quantitative high-throughput spike [S] protein ELISAs [enzyme-linked immunosorbent assays], live viral neutralization assays and T-cell assays to quantify antibody and cellular immune responses to gain a better understanding of which elements of the immune response are necessary for protection and to help define immune correlates of protection. Virus variation may prove challenging to the interpretation of these immune responses, and these responses may vary by different age groups. We are also seeking second-generation vaccines that may elicit a greater breadth of immune response, including mucosal immunity and induction of T-cell responses against a broader set of viral antigens beyond SARS-CoV-2 S protein.

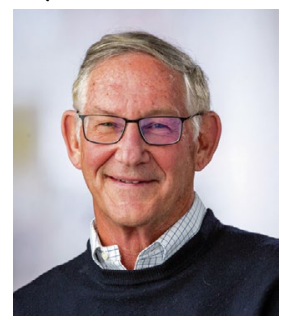

Larry Corey: Only vaccine trials themselves with assessment of some threshold of immune protection will define what type and levels of vaccine-induced immunity are Larry Corey, Fred Hutchinson Cancer Research Center. needed to achieve protection from either SARS-CoV-2 infection or disease. Natural infection

cohorts can give potential targets to consider to achieve immunity, but all the initial vaccine candidates at the moment are designed to induce immune responses to the $S$ protein and not to the panoply of antigens associated with natural infection. Persons with severe disease tend to develop antibodies sooner and at a higher magnitude than those with mild disease - an observation made with both SARS-CoV-1 and SARS-CoV-2.

John Moore: Immunity to the common cold coronaviruses definitely wanes with time, such that people become reinfected after about a year (both naturally and in long-ago virus-challenge experiments). Antibody titers to SARS [severe acute respiratory syndrome] and MERS [Middle East respiratory syndrome] also decrease significantly over time, but very little was ever learned about immune memory to those viruses, and $\mathrm{T}$-cell responses were rarely studied ${ }^{1}$.

It's going to be very important to monitor convalescent COVID-19 patients, particularly those with mild disease and low-level antibody titers, to see if and when reinfections do become more common. At the moment, we're hearing only rare and sometimes controversial anecdotes. What's the duration and extent of immune memory? What antibody and T-cell measurements are associated with any reinfections that are well documented? 
There is an increasing amount of data on T-cell responses to the OWS [Operation Warp Speed] vaccines, although the balance of evidence is strongly toward neutralizing antibodies being more important for protection. I would like to see much more data on mucosal responses, particularly IgA [immunoglobulin A]. It's pretty surprising there's so little known so far.

My assumption is that vaccine immunity to SARS-CoV-2 may not be long-lasting (a year or two?) and that booster immunizations might be needed if the virus is still circulating. This, of course, remains to be proven.

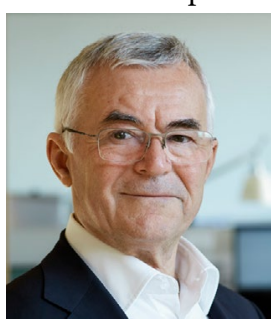

Rino Rappuoli,

GlaxoSmithKline.

Rino Rappuoli:

The work on SARS

and MERS vaccines

showing good

protection in animal models increases our confidence that vaccines for SARS-CoV-2 will be possible. The data suggest that serum neutralizing antibodies are

sufficient to protect from infection. The importance of serum neutralizing antibodies is also confirmed by the experience of treating COVID-19 patients with plasma from convalescent patients, showing impact on clinical disease and on viral load. Several studies on human monoclonal antibodies derived from memory B cells from COVID-19 convalescent patients also confirm in animal models that neutralizing antibodies protect from infection and improve the disease outcome.

\section{What types of preclinical studies are needed to fill gaps in our knowledge of SARS-CoV-2 immunity and galvanize progress in vaccine design, safety and efficacy?}

G.A.: Unquestionably, each vaccine should be tested in non-human primate [NHP] models, where the virus shows different levels of pathogenicity. Thus, rhesus macaques show some lung pathology, but largely experience asymptomatic infection, whereas other monkey species appear to experience more severe disease. Given that the outcome in most vaccine studies will be severity of disease, it is essential to perform deep immunological investigations across animal species with different degrees of disease severity to preempt any potential harm caused by vaccines. Moreover, the identification of mechanistic correlates of immunity that can be defined in passive transfer studies in primates or other animal

models would point to specific endpoint immunological assays that could be incorporated into phase 3 testing and help with vaccine licensure.

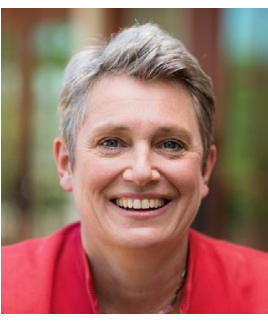

Kate Bingham., SV Health Managers and UK Vaccines Taskforce. Credit: Tom Kates

[for COVID-19]; as do Syrian hamsters and ferrets. The typical rhesus macaque models used in SARS-CoV-2 studies involve young animals that recover from infection on their own, mirroring that seen in infections of (young) humans. NHP models have been used to show antibody-dependent enhancement (ADE) pathology using the adjuvant alum, and so data from NHP models can form part of the preclinical safety package for regulators.

Given the wealth of immunological, and ultimately efficacy, data that are being generated in clinical trials, preclinical models will be far less relevant than human efficacy clinical data. The problem with lab animals is that they have had no external exposure to disease. NHP data are not likely to have substantial impact on the efficacy decisions of which vaccines to use. The best thing to do is to study the immune response in patients who have been infected with the virus and recovered and try to mimic this with a vaccine. This means deep study of the $\mathrm{T}$ - and B-cell responses in humans, in response to both vaccine and the natural infection.

L.C.: Mice and NHP animal models show that high antibody titers are more likely to lead to increased vaccine-induced protection. Passive transfer studies suggest neutralizing antibodies can protect the lung and perhaps, in high titers, the upper airway (likely partially). The human efficacy studies for SARS-CoV-2 in the United States are large (30,000 persons) and hence make it difficult to assay cell-mediated correlates of protection, but are designed to evaluate correlates of protection for neutralizing and binding antibody assays. Hopefully, functional antibody responses to the $S$ protein will be associated with clinical effectiveness.

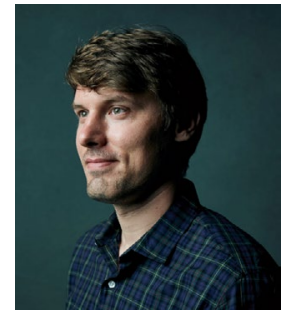

James Dahlman: One key study would be to further optimize mRNA vaccines in non-human primate models by varying the immunogen and the lipid nanoparticle (LNP) carrying the mRNA. It is James Dahlman, Georgia Institute of Technology. encouraging that the first-generation mRNA vaccines both demonstrated

safety in early clinical trials. Using these first vaccines as a starting point, next-generation SARS-CoV-2 vaccines, which may be needed if the virus persists over the coming years, could be improved.

J.M.: I would like to see animal, particularly macaque, challenge studies [in which viral inoculation is] delayed for substantial periods of time after the peak of the vaccine-induced antibody response. There is a single preprint showing protection (viremia reductions) after a delayed challenge (77 days) in DNA-vaccine-immunized macaques. That kind of study needs to be more widely performed and extended in time, to provide us with a feel for the duration of protective immunity. Ideally, we would have a disease model, and not just the existing mild infection-then-clearance models (macaques, various small animals, transgenic hACE2 [human angiotensin-converting enzyme 2] mice). R.R.: Although we do not have perfect animal models, rhesus macaques, hamsters and mice expressing the hACE2 receptor are available and provide good information to make decisions before going to humans. Clearly, humans remain the best model. In vitro studies on mucosal IgGs and IgAs may also be important, especially to understand protection in the upper respiratory tract and impact of vaccines on transmission. Finally, studies on T-cell immunity will be important, especially as some of the vaccine platforms, such as RNA and viral vectors, induce $\mathrm{CD} 8^{+} \mathrm{T}$ cells in addition to neutralizing antibodies.

What have we learned from previous vaccines, and how well have results from non-human primate studies translated to humans?

R.R.: The beautiful work done by Jason McLellan to stabilize the $S$ protein of MERS coronavirus in the prefusion conformation ${ }^{2}$ provided the knowledge to up-front engineer SARS-CoV-2 S protein in the prefusion-stabilized form. This information has been the basis for most of the COVID-19 
Table 1 | SARS-CoV-2 vaccines in late-stage clinical development

\begin{tabular}{l} 
Sponsor \\
\hline Beijing Institute of Biological Products, \\
Sinopharm \\
Wuhan Institute of Biological Products, \\
Sinopharm \\
Sinovac Biotech, Instituto Butantan, \\
Bio Farma \\
University of Oxford, Oxford Biomedica, \\
Vaccines Manufacturing and Innovation \\
Centre, Pall Life Sciences, Cobra Biologics, \\
Halix, Advent, Merck, Serum Institute of India, \\
Vaccitech, Catalent, AstraZeneca, IQVIA \\
Moderna, NIAID, Lonza, Rovi, Medidata, \\
Bioqual
\end{tabular}

Gamaleya Research Institute of Epidemiology and Microbiology

CanSino Biologics, Beijing Institute of Biotechnology, National Research Council of Canada

BioNTech, Fosun Pharma, Pfizer

Institute of Medical Biology, Chinese

Academy of Medical Sciences

Anhui Zhifei Longcom Biopharmaceutical; Institute of Microbiology, Chinese Academy of Sciences

CureVac

Zydus Cadila

vaccines in development. In theory, the RBD is also sufficient to induce good titers of neutralizing antibodies, but it requires engineering of the protein in dimers or nanoparticles to be an efficient immunogen. G.A.: The translation of NHP studies to humans has only emerged recently, as our understanding of the genetic and immunologic parallels and differences have begun to emerge. It is essential that these studies be performed. In HIV vaccine design, emerging data have shown similar correlates of protection in NHP and humans, demonstrating the power of the NHP model for the iterative improvement of vaccine strategies, as well as a model in which mechanistic correlates of immunity

\section{Vaccine type (product)}

Inactivated virus

Inactivated virus

Inactivated virus (inactivated

SARS-CoV-2 plus alum; CoronaVac; formerly PiCoVacc)

Non-replicating viral vector (chimpanzee adenoviral vector encoding S protein; AZD 1222; formerly ChAdO $\times 1$ )

RNA-based (nucleoside-modified mRNA vaccine encoding codon-modified S protein encapsulated in ionizable LNPs, containing distearoyl phosphatidylcholine, cholesterol and polyethylene glycol lipid)

Ad5 and Ad26 with a coronavirus gene, administered separately

Non-replicating viral vector (Ad5 vector Phase 3 encoding S protein)

RNA-based (mRNA vaccine expressing codon-optimized, nucleoside-modified mRNA that encodes trimerized RBD in ionizable cationic LNPs containing phosphatidylcholine, cholesterol, polyethylene glycol lipid)

Inactivated virus

Protein subunit (adjuvanted recombinant protein $\mathrm{RBD}$ dimer)

Protamine-complexed mRNA vaccine expressing undisclosed SARS-CoV-2 protein

Electroporated DNA vaccine encoding undisclosed SARS-CoV-2 protein

stage

Phase 3

Phase 3

Phase 3

Phase 3

Phase 2

Phase 2

Phase 2

Phase 2

\section{Clinical Trial ID}

Phase 3 ChiCTR2000032459, ChiCTR2000034780

ChiCTR2000031809, ChiCTR2000034780

NCT04456595, NCT04352608, NCT04383574, 669/UN6.KEP/EC/2020

NCT04324606, EudraCT 2020-001072-15, EudraCT 2020-001228-32, NCT04400838, ISRCTN89951424, PACTR202006922165132 NCT04283461, NCT04405076, NCT04470427

NCT04530396

NCT04313127, ChiCTR2000030906, ChicTR2000031781, NCT04398147, NCT04341389, NCT04456595

Phase $2 / 3$

EudraCT 2020-001038-36, NCT04368728, ChiCTR2000034825

NCT04412538, NCT04470609

NCT04445194, NCT04466085

NCT04515147

CTRI/2020/07/026352

can be defined. But given the differences in antibody immunology across antibody subclasses/isotypes and in Fc receptors, precise mechanistic correlates of humoral immunity may not be fully defined in the animal model.

K.B. Human challenge models in flu revealed critical information and data about the infectivity of subjects before their showing symptoms, and this was only discovered through tightly controlled human challenge studies. It will be important to explore the ethics and practicalities of human challenge studies for the controlled testing of new COVID-19 vaccines, where subjects receive the vaccine before being [deliberately] infected by the virus. This will require close working with MHRA [the UK's Medicines and Healthcare Products Regulatory Agency] and ethicists to secure regulatory approval, which will include robust protocols to rescue subjects exposed to virus.

J.M.: There's always a concern [about translation] .... In my field of HIV vaccines, there has been a strong tendency over the years to emphasize 'good results' in macaques when it comes to pushing on to human trials, but to dismiss 'bad results' on the grounds that "it's just an animal model."

We also know from the HIV/simian immunodeficiency virus/simian human immunodeficiency virus (and no doubt other) vaccines that it's easier to protect 
against minimally replicative/pathogenic viruses than it is against their more virulent counterparts. Hence, there is a nagging concern that we could be seeing protection against SARS-CoV-2 replication and disease in macaques that won't translate to protecting humans. Time will tell ....

There's also no universal standard for how macaque studies are carried out or analyzed. For example, it looks like the Johnson \& Johnson vaccine experiment ${ }^{3}$ used a rhesus macaque challenge virus dose that's $\sim 10$-fold lower than what was done in the [US National Institute of Allergy and Infectious Disease (NIAID)] Vaccine Research Center/Moderna study ${ }^{4}$. And variation in the challenge stock/dose/route is generally true across the macaque challenge studies. How do those differences affect the outcome? It's also very difficult, and sometimes impossible, to cross-compare the antibody and T-cell responses in the various studies because the assays and data presentation methods are too different. There sometimes seems to be an emphasis on finding ways to report 'big numbers'.

Considering the diverse range of vaccines under development, which are most likely to induce protective antibodies with an acceptable safety profile?

G.A.: This is a loaded and difficult question. I do not think it is challenging to induce neutralizing RBD [receptor binding domain]-specific antibodies against SARS-CoV-2. These antibodies seem to be sufficient to provide protection against viremia in the lung. Moreover, there are many vaccine platforms that appear to make antibody responses that last for several years. It is therefore likely that many types of vaccine are feasible against SARS-CoV-2. However, whether distinct platforms will drive similarly long-lived immune responses remains unclear, but will emerge from the multitude of phase 3 trials that will take place in the race against this virus. These data will not only be informative for SARS-CoV-2 vaccine development, but will have a tremendous impact on how we think of vaccines for the future.

K.B.: SARS-CoV-2 vaccines in development (including viral vectors, nucleic acid, protein and inactivated whole-virus vaccines) have generally been selected on strong preclinical data in a variety of species that show good immune responses (including antibody and T-cell responses) and in most cases protective immunity in preclinical models. Protein antigens as vaccines often have insufficient immunostimulatory capabilities on their own so are usually coadministered with adjuvants, such as GlaxoSmithKline's ASO3 or Dynavax's CpG 1018, to enhance

their immunogenicity. Inactivated whole-virus vaccines are immunogenic and also dosed with adjuvants to boost the immune response. No RNA [or DNA] vaccine has been approved for any human disease at this point, but the standardized approach enables agile development of new products in response to rapidly mutating viruses (albeit that SARS-CoV-2 does not currently appear to accrue mutation at a high rate). We are yet to see how the different SARS-CoV-2 vaccines compare in terms of which one confers the most long-lasting immunity.

L.C.: Theoretically, all the vaccine platforms - protein antigen, RNA or DNA plasmids, or viral shuttle vectors - are capable of eliciting protective antibodies. Evidence of this in NHP is seen with RNA vaccination, recombinant Ad26 [adenovirus serotype 26 ] vectors expressing SARS-CoV-2, and protein antigens. Whether these data in small-animal and NHP models translate to human infection and disease remains to be determined. I am cautiously optimistic and hopeful.

J.D.: It is too early to know whether a protein antigen or a nucleic acid expressing an antigen is more likely to induce protective antibodies. Given the need to create a safe vaccine as soon as possible, it is unlikely we will have conclusive evidence before the first SARS-CoV-2 vaccines are approved. In addition, vaccine potency depends on many interacting variables, such as the immunogen, its glycosylation pattern, its presentation, and the presence of specific adjuvants. As a result, it is unlikely that one vaccine class (for example, mRNA) will always be better than another (for example, protein antigen). That said, studies over the next few years will likely provide the answer for the first generation of $\mathrm{CoV}-2$ vaccines; these data will inform the design of improved $\mathrm{CoV}-2$ vaccines or vaccines against future coronaviruses.

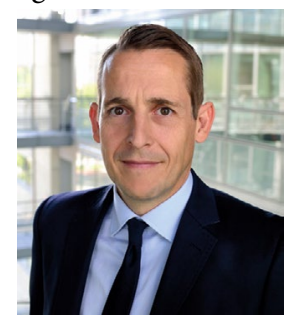

Nick Jackson, CEPI.
Nick Jackson: In the absence of a correlate of protection, it is necessary to work on first principles and glean insights from immunological investigations in the MERS and SARS-CoV-1 fields. As such, the field is aligned that a specific, robust, rapid-onset and long-lived humoral neutralizing response could protect vaccinees against SARS-CoV-2 disease. Cell-mediated immune responses will likely supplement neutralizing antibody protection.
A plethora of early clinical data is emerging on all the different platforms, but an international standard with which to coordinate assays across laboratories is not yet available. It is also unclear how the level of assay variability of these assays impacts the interpretation of data. Thus, it remains impossible to make comparative assessments on the basis of available phase 1 data. Groups have attempted to calibrate assays by including convalescent sera as an internal control. In most cases, elicited responses appear to be in the range of convalescent sera. Given that efficacy trials are ongoing and expected to report interim results toward the end of 2020 and into 2021, the question of superior immunogenicity is arguably now superseded by point estimates of efficacy to prevent virologically confirmed disease. In totality, it is expected that the array of vaccine platforms being developed will likely result in a range of different estimations of efficacy.

J.M.: An adjuvanted subunit protein will surely be the strongest immunogen for inducing neutralizing antibodies. Recent macaque and human ${ }^{5}$ immunogenicity data from Novavax fully support that statement. The single-dose adenovirus vectors don't do well, but in some cases there are improvements when a booster dose is given ${ }^{6-8}$.

\section{What aspects are important in the context of a goal of immunizing the largest fraction of the world's population?}

G.A.: Immunogenicity, safety and tolerability are the most critical aspects for selecting globally relevant vaccines. Some vaccine platforms may be more immunogenic, but may induce more adverse reactions. Moreover, some platforms will drive different responses in children than adults. Furthermore, some platforms may not work well in immunocompromised patients or the elderly, due to their unusual and often less potent immune systems. Thus, testing is required across all populations to make the careful decisions for vaccine development. It is plausible that different solutions will work for distinct populations. Thus a single emergency authorization should not be regarded as global solution. K.B: No vaccine, irrespective of format, should be administered anywhere in the world without a comprehensive safety dataset, approved by regulators. Prevalidated platforms and the adenoviral vector formats developed by Oxford (ChAdOx1; adenovirus serotype 6 vector) and Johnson \& Johnson (Ad26 vector) can be engineered rapidly to include the relevant SARS-CoV-2 S protein sequence and scaled up quickly to produce hundreds of millions of doses of vaccine 
relatively cheaply. Given the flexibility of their adeno-vaccine format, Oxford was able to start dosing clinical trial volunteers in April 2020, having only received the SARS-CoV-2 sequence in January. If these vaccines are proven safe and effective, AstraZeneca and Johnson \& Johnson each seem set for global distribution in 2021 to produce $\sim 2$ billion doses of ChAdOxl and Ad26, respectively.

The vaccine formats with the greatest potential for speed of scale up are RNA-based platforms, such as those from BioNTech, Curevac and Moderna. These can be made quickly because they use synthetic processes, without culture or fermentation, once the DNA template itself is made. Self-amplifying RNA (saRNA) vaccines, such as that being developed by VacEquity Global Health in Imperial College, have potentially greater advantages of manufacturing since the dose required for a saRNA vaccine is substantially lower than for other mRNA vaccines. But mRNA is naturally unstable and needs to be formulated in LNPs and stored frozen at below $-20^{\circ} \mathrm{C}$, which will complicate the global shipping and clinical use of RNA vaccines, especially in the developing world. Although the relative cost of mRNA vaccines is currently high, I expect these costs to reduce once the mRNA vaccine technology is optimized and fully scaled.

Recombinant-subunit vaccines combined with adjuvants are relatively cheap to make, easily scaled, are widely used as vaccines throughout the world and are reasonably stable and so easier to manage for global distribution. There is widespread experience with these types of vaccines and supply chains are well established. However, they depend on biological processes and so have been slower to manufacture and develop, currently running about 6 months behind the adeno and mRNA leading vaccine candidates in development.

Whole inactivated virus-based vaccines are well understood and effective vaccine formats, but live virus Biosafety Level 3 (BSL-3) manufacture has not yet been scaled to volumes suitable for global distribution. L.C.: I personally think a lot of different platforms are both desirable and necessary to achieve global immunization. Desirable because of the diversity of the population that needs to be immunized and necessary because there are manufacturing and logistical issues in rapidly making vaccine accessible to the vast population (seven billion people) who need to be vaccinated. Women of child-bearing age, children, older adults and pregnant women are unlikely to be best managed and vaccinated with just one vaccine. RNA vaccination has little experience in children and pregnant women - no evidence they are dangerous, but little evidence for proven safety. The proteinadjuvant platforms have long experience in children. The Johnson \& Johnson Ad26 vector does have experience in pregnant women and women of child-bearing age and has excellent manufacturability. In the end, a diversity of platforms is best economically as well as to allow more rapid distribution throughout the world.

The one platform I think should not be pursued is that using Ad5 [adenovirus serotype 5] vectors. Ad5-based vaccines that lack HIV envelope have been associated with an increased risk of HIV-1 acquisition. I worry that Ad5 COVID-19 vaccines [for example, CanSino's] could duplicate that risk and feel that Ad5 vector should be used with caution and the risk of increased HIV acquisition should be a factor in its assessment.

N.J.: Historically, widespread successful immunization campaigns have required high volumes of vaccine drug product; thermal stable formulations that don't require a complex cold-chain distribution; a well characterized, simple immunization device; and low cost of goods. The current COVID-19 vaccine frontrunners have a variety of limitations. In the case of mRNA, the productivity of manufacturing remains low and cold-chain requirements will impose significant challenges for immunization campaigns in certain regions. As a result, the cost of goods is expected to be significant. Viral vectors are expected to yield higher annual volumes, in the hundreds of million doses range. However, certain vector platforms have thermal stability limitations.

Based on currently available data for protein vaccine approaches, it is expected that the annual manufacturing capacity could reach a billion or more doses, and more robust thermal stable formulations are being advanced. It is therefore reasonable to predict that protein-based vaccine approaches will ultimately reach a larger fraction of the world's population assuming other factors such as efficacy, safety and equitable access are equivalent.

R.R.: The key question will be which vaccines are effective in providing immunity against severe disease, infection and transmission - and how long that immunity is going to last. Although published data provide safety and efficacy data on all the different vaccine platforms, because a variety of non-standardized neutralizing assays were reported, direct comparison is impossible. But my feeling is that vaccines based on recombinant proteins

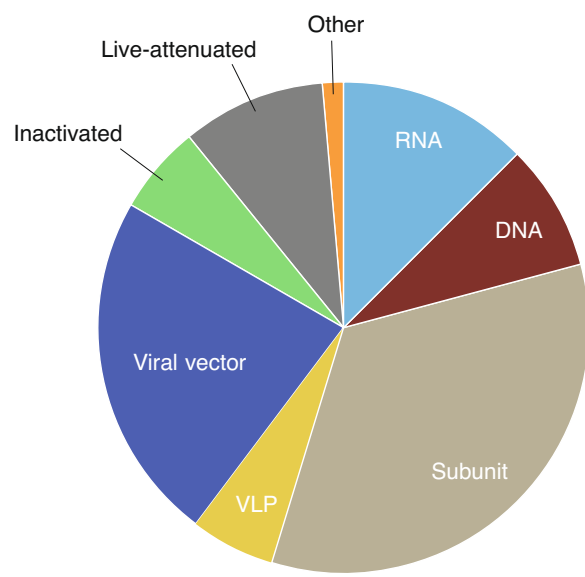

COVID-19 vaccine candidates under development. Adapted with permission from ref. ${ }^{17}$, Springer Nature.

and adjuvants may induce the highest level of neutralizing antibodies, followed by RNA vaccines and viral vectors.

The other important question will be vaccine safety. Viral vectors and RNA vaccines have never been used in millions of people, and we need to look carefully at this. J.M.: There are practical concerns about the mRNA vaccines, given their thermal instability. [Moderna's vaccine needs to be kept at $-20^{\circ} \mathrm{C}$, whereas the Pfizer/ BioNTech vaccine needs to be at $-70{ }^{\circ} \mathrm{C}$.] A mass rollout may be problematic, given the apparent need for them to be shipped on dry ice and stored in ultra-cold freezers until just before use. But each of the various designs may end up playing a role in different settings, provided safety and efficacy is proven in the longer term in each case.

For nucleic acid vaccines, what is known about the effects of sequence/ modifications, mode of vaccine delivery and host genetic factors on protein immunogen expression?

G.A.: For RNA vaccines, there are likely to be massive differences in the durability and level of protein expression depending on the cell type and organ where LNPs are delivered. The level of protein, its glycosylation, other post-translational modifications, and protein turnover will all vary. Conversely, viral vectors have tropism and take advantage of specific cell type protein production machinery, as well as antigen-presentation capacities that may have a critical effect on the quality and potency of the induced immune responses.

Novel chemical modifications to RNA and DNA vaccines may profoundly influence the quality of the immune responses induced by these novel vaccine 
platforms. Nucleic acids not only carry the critical vaccine-message, but can also trigger immunity through pattern recognition receptors themselves. Thus, these emerging platforms may hold interesting and unexpected possibilities for immune programming that go beyond the vaccine insert.

Finally, one important consideration is efficiency of vaccine delivery. While viral vectors take advantage of millennia of evolution that endow viruses with the ability not only to target a particular cell type, but also to release viral nuclear material in a protected manner to the intracellular compartments where it may replicate, protein- and nucleic acid-based strategies are somewhat untargeted. Large amounts of protein-based vaccines are likely degraded, but some proteins are taken up and processed by antigen-presenting cells and represented to the immune system. Conversely, nucleic acid-based vaccines not only must get taken up by cells, but also must breach the endosomal compartment and release RNA into intracellular compartments that can drive translation for RNA vaccines, or even make into the nucleus to drive transcription for DNA vaccines. Thus, each platform may access the cellular machinery in very different ways that may alter antigenicity and immunogenicity.

K.B.: Optimizing protein expression is an essential part of vaccine development for any vaccine. One of the advantages of RNA vaccines is that this optimization is relatively easy. Clearly, the LNP is an integral part of any RNA vaccine and selection of an optimal lipid composition is essential for delivery. The mRNA cap sequences also need careful optimization so that the appropriate codons can be chosen to make the appropriate amino acids to ensure good expression levels. Future work will need to optimize the LNP to stabilize the mRNA product, minimize any degradation and avoid the need for a challenging cold chain in distribution.

I am not aware of data on changes in expression levels from RNA or DNA in different demographic populations, and I am not sure there would be expected to be any substantial differences.

J.D.: The expression level of mRNA vaccines delivered into cells by LNPs is influenced by the mRNA structure as well as LNP chemical composition. With respect to the mRNA, changes to its cap, 5' UTR [untranslated region] and 3' UTR and the inclusion of chemically modified nucleotides can increase the amount of protein produced per unit mRNA that enters the cell. These changes are additive and sometimes synergistic; an mRNA with a potent UTR can be further improved by including chemically modified bases. Finally, one underappreciated trait is the purification process used after mRNA is transcribed. Purification that reduces double-stranded RNA byproducts leads to higher quality mRNA.

The chemical composition of the LNP also potently affects mRNA delivery. LNPs are typically composed of four components: ionizable lipids, poly(ethylene glycol)-lipids, cholesterol and helper lipids. All four components work together to influence how efficiently LNPs deliver mRNA into cells. As a result, many are searching for an LNP mixture that maximizes protein production.

Finally, although evidence is still emerging, I am increasingly convinced that protein production requires us to understand cell signaling. Recent papers have shown that mTOR, $\mathrm{PIP}_{3}$ [phosphatidylinositol 3,4,5-trisphosphate] and TLR [Toll-like receptor] signaling can influence nanoparticle-mediated mRNA delivery $^{9-11}$. I would therefore hypothesize that certain cell 'states' are more amenable to mRNA production after LNP delivery than other cell states.

As yet, mRNA and DNA vaccines have not been tested at the scale of human populations, so we don't know about variation at this level due to demographics, genetic variation or environmental factors. It is even difficult to use preclinical data to guess; I am unaware of comprehensive datasets evaluating how mRNA or DNA vaccines work in non-human primates as a function of age or disease state. However, there may be an interesting opportunity to couple large biobanks (for example, the UK Biobank, which sequences samples from hundreds of thousands of people) with SARS-CoV-2 clinical data to see if there are genetic determinants that alter the efficacy of these vaccines.

N.J.: The level of protein expression and the subsequent elicited immune response are significantly impacted by the critical quality attributes (CQAs) of mRNA ${ }^{12} .5^{\prime}$ capping efficiency, 3' UTR, poly(A) tail properties, coding sequence modification and the purity of the construct can all have a major impact on expression. In addition, the ability of the LNP formulation to successfully deliver the mRNA construct into the cytoplasm of the target cells is of paramount importance. To date, head-to-head comparisons of these CQAs in controlled clinical trials are lacking, as is the assessment of self-amplifying versus non-replicating constructs.

For viral vector vaccines, how much of a limitation is pre-existing immunity?

K.B.: There are two issues here: pre-existing immunity to the vector and pre-existing immunity (if any) to the antigen (that is, the vaccine sequence itself). Pre-existing immunity against Ad5 (a human cold virus) is widespread in the population, thus potentially hampering its clinical use as a vaccine vector (at least when delivered intramuscularly, since intranasal delivery may bypass pre-existing immunity). Recent CanSino clinical data showed that people who already had immunity to the Ad5 backbone of the vaccine had a reduced immune response to the COVID-19 S protein. Instead, simian adenoviruses (ChAdOx1 and Ad26) have been developed since they have minimal seroprevalence in humans. We don't yet know about pre-existing immunity to the SARS-CoV-2 S protein or other SARS-CoV-2 proteins, but pre-existing immunity to this could have all sorts of positive and negative implications.

N.J.: It has been demonstrated in several clinical trials that pre-existing immunity to certain viral vectors can reduce immunological potency. However, some viral vectors, such as measles-vectored candidate vaccines, do not appear to be impacted by varying levels of pre-existing immunity.

J.M.: For the CanSino Ad5 vector vaccine, being developed in China, pre-existing immunity does seem to be a substantial problem, which was entirely foreseeable based on what's known from studies in, for example, the HIV vaccine field. There are no signs that such problems are affecting the other adenoviral vectors, Ad26 and ChAdOx1, which were designed to overcome that particular problem.

\section{Why are comparatively few inactivated or live-attenuated vaccines being devel- oped against SARS-CoV-2?}

G.A.: These are older approaches that have been replaced by super-high-end, cutting-edge, highly flexible vaccine platform technologies that are more controllable, predictable, safer, simpler and manufacturable.

K.B.: Developing a safe live-attenuated vaccine (LAV) takes a long time and has many more safety hurdles than protein, viral or mRNA/DNA-based vaccines. Regulatory-approved BSL-3 containment facilities are required to grow live virus for whole inactivated vaccines, and BSL-3 manufacturing capability in is very limited throughout the world.

N.J.: In China, there are actually several inactivated vaccines being developed, and one is currently undergoing phase 3 clinical assessment. Their limited application elsewhere may reflect concerns for the low levels of manufacturing yields, potentially lower immunogenicity in the elderly, 


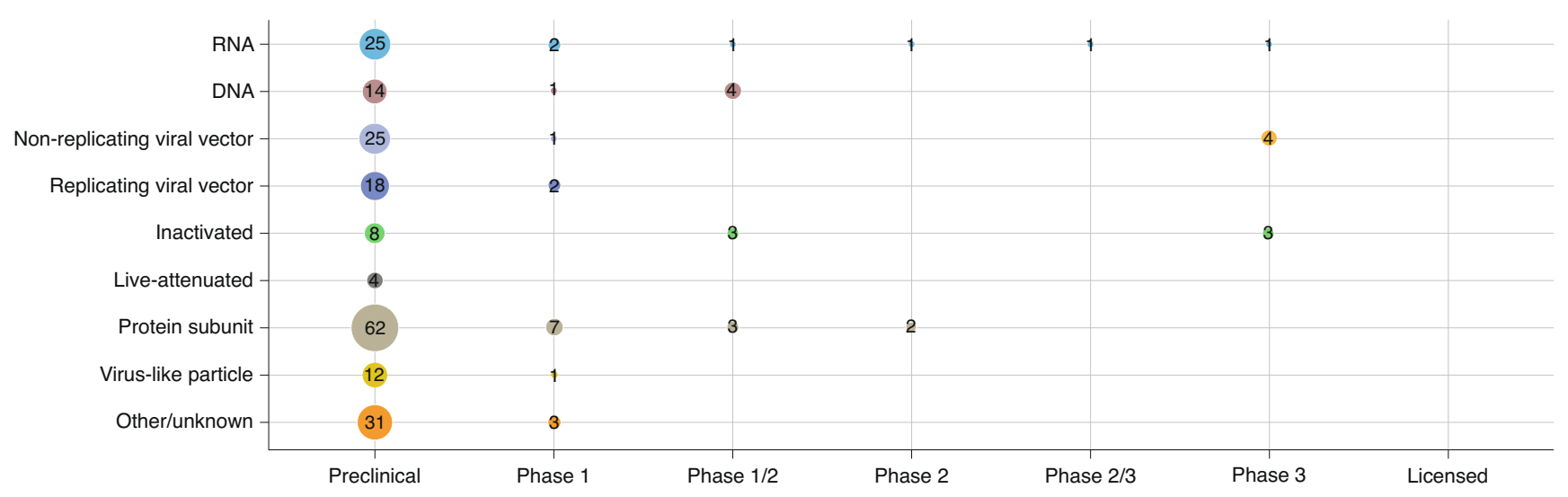

Stages of development of COVID-19 vaccine candidates. Adapted with permission from https://vac-Ishtm.shinyapps.io/hcov_vaccine_landscape/, Vaccine Center at the London School of Hygiene and Tropical Medicine.

challenging manufacturing requirements and putative concerns over safety. LAVs are the basis of many successful, highly efficacious vaccines. However, the time to develop a LAV is typically protracted to find the right level of attenuation while maintaining sufficient immunogenicity. For these reasons, the field has few LAV currently in development.

J.M.: I suspect the simple answer is that the major American and European vaccine development companies have 'moved on' from those earlier generation concepts. The Chinese, however, have retained a substantial production capacity for killed-virus vaccines and are using it for COVID-19. For attenuated vaccines and RNA viruses, there's always a concern about reversion to virulence, and that may be a factor here.

\section{What is the optimal vaccine} immunogen against SARS-CoV-2?

G.A.: The $S$ protein. There are multiple emerging sites of neutralizing antibody vulnerability across the $S$ protein compared, beyond the RBD. In addition, extra neutralizing antibody functions (antibody-dependent cellular cytotoxicity, Fc-mediated phagocytosis, complement activation etc.) may all be critical for protection and can be targeted along the whole surface of the $S$ protein.

K.B.: The current data suggest that antibodies against $\mathrm{S}$ protein are neutralizing. In theory, both $S$ protein and its RBD domain should induce an immune response. The VTF is exploring second-wave vaccines incorporating other antigens to be combined with the $\mathrm{S}$ antigen, but these are not as advanced. LAV vaccines have multiple potential antigens. The T-cell responses when correlated to protection will tell us more about which viral proteins are required for protective cell- and antibody-mediated immunity.

N.J.: One way to answer to this is to look at the immune response upon natural infection and the targets of functional antibodies. These are largely directed to the $\mathrm{S}$ protein and, within it, to the RBD, the rationale being that blocking the RBD would prevent the virus from adhering to the receptor, a first step toward infecting a cell. A second requirement is to stabilize the $S$ protein in a prefusion state, which is metastable in nature and in its trimer native form. Mutations within the $\mathrm{S}$ protein can actually stabilize it in a prefusion form and make it a stronger immunogen than the native $S$ protein of the virus.

J.M.: I would choose RBD over S protein, which is why I prefer the Pfizer/BioNTech mRNA vaccine design to Moderna's. For one thing, there is less chance of inducing non-neutralizing antibodies that may be problematic in some scenarios. If T-cell helper responses are being sought, including (for example) the $\mathrm{N}$ [nucleocapsid] protein could be helpful, but not for inducing neutralizing antibodies directly.

\section{How important is proper glycosyla-} tion and proper folding of the immunogen likely to be for vaccine efficacy?

G.A.: Glycans may influence, but are not critical for, RBD immunogenicity. Conversely, glycosylation on $\mathrm{S}$ will be critical, particularly given the unusual placement of glycans on the post-cell-fusion state of the protein. While emerging data point to the importance of glycan microheterogeneity at some sites for immunogenicity in HIV vaccine development, it is unclear whether glycan structures will alter SARS-CoV-2 vaccine immunogenicity.

All viral-vectored and nucleic acid-based approaches leverage 'self' glycosylation [as they are expressed in the recipient's cells rather than an ex vivo system]. Recombinant antigen approaches, like Novavax's, will also have mammalian glycosylation [courtesy of Chinese hamster ovary ( $\mathrm{CHO}$ ) cell line expression]. The baculovirus- and plant-based recombinant systems may have some immunogenicity issues; on the other hand, their altered glycosylation patterns may enhance C-type lectin-driven processes that deliver immunogen to immune sensors as pattern recognition signals, so these type of recombinant vaccines may be self-adjuvanting.

Interestingly, host-cell glycans play a critical role for early viral attachment, before RBD attachment to ACE2. This early $S$ interaction with cellular glycans helps the virus attach in a likely arduous respiratory environment, highlighting the importance of glycosylation both for vaccination and also in the context of natural infection/ disease pathogenesis.

K.B.: Glycosylation is important for vaccine efficacy since it may affect which cells the SARS-CoV-2 virus can infect and could shield some epitopes from antibody neutralization. Recent data show that each protomer in the trimeric $S$ protein has 22 glycosylation sites. For SARS-CoV-2 vaccines, the role of glycosylation in the immune response is not yet understood. In theory, production of the protein in mammalian or human cells should give rise to the most natural antigens with the right glycosylation patterns. It is clear that the conformation of the $\mathrm{S}$ protein is important, and the appropriate conformations have been designed into several of the vaccine candidates (for example, Moderna's encoded immunogen has the RBD binding arms up). J.M.: If you had asked me before immunogenicity data emerged, I would have said these issues were very important 
based on my experience in the HIV-1 envelope glycoprotein arena (equivalent to the SARS-CoV-2 S protein). However, that appears to be not (much) the case with the $S$ protein and, in particular, its RBD. Some animal model studies have shown that high neutralizing antibody titers are rapidly induced almost irrespective of how the RBD is produced and what adjuvant is used ${ }^{1}$, including at least one (complete Freund's) that is pretty harsh on protein conformation. The RBD is also relatively glycan free; insect-cell-produced S proteins, including the Novavax clinical candidate ${ }^{5}$, seem to be quite immunogenic for anti-RBD neutralizing antibodies, despite their 'atypical' glycans when delivered with or without adjuvants.

\section{What types of adjuvant are likely to be most effective in eliciting not only neutralizing antibodies but also the right type of helper responses?}

K.B.: All available adjuvants have been designed to be antigen dose sparing along with producing strong antibody and cellular responses in most cases. Until the combinations are tested with COVID-19 vaccines, it is not possible to say which combination makes for a safer, more efficacious vaccine. There have been safety issues with alum in the past, and we need to look at all aspects of preclinical and clinical immune response to ensure safety is managed appropriately. In addition, emerging data suggests previous exposure to adjuvants, such as AS03 in Shingrix [zoster vaccine recombinant, adjuvanted], may be important in providing protection against COVID-19. N.J.: For protein-based vaccine candidates, the adjuvant brings a priori three things to the antigen(s). First, it stimulates and increases the immune response; second, it potentially reduces the amount of antigen needed; and third, it orients the immune response toward a T-helper $1\left(\mathrm{~T}_{\mathrm{H}} 1\right)$ antibody response. Adjuvants known to orient the immune response toward $\mathrm{T}_{\mathrm{H}} 1$ would therefore be prioritized, while those that orient the response toward a $\mathrm{T}_{\mathrm{H}} 2$, such as alum, should be cautiously evaluated. A concern is the phenomenon known as the ADE, which occurs when antibodies enhance viral infection, possibly by increasing viral entry or promoting inflammation and tissue injury. This has been observed with other, non-COVID-19 vaccine candidates formulated toward a $\mathrm{T}_{\mathrm{H}} 2$ response and can be evaluated in animal models.

J.M.: Adjuvants are only being used, so far, for the protein subunit vaccines. And, as noted above, a range of them appear to be effective, at least when used with the RBD. But what matters most is that only a very

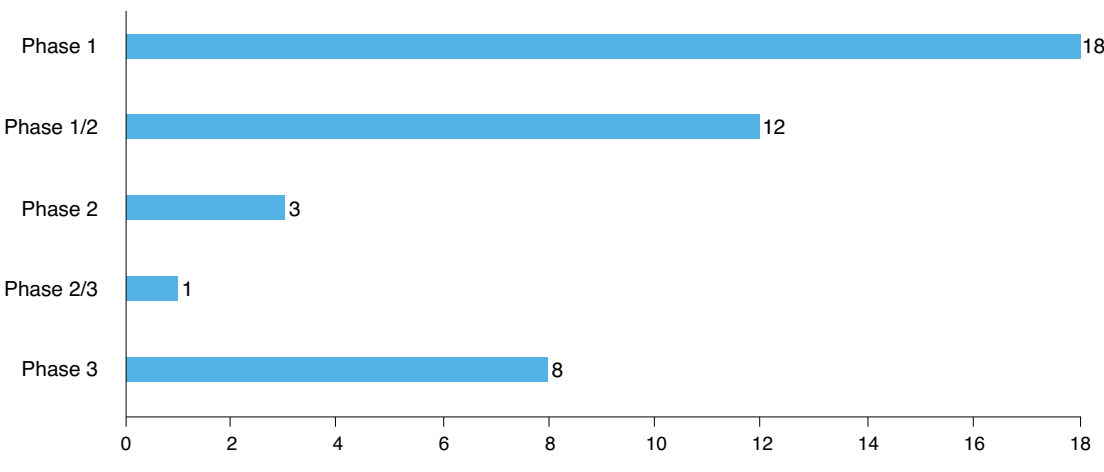

COVID-19 clinical trials by stage of development. Source: London School of Hygiene and Tropical Medicine.

few adjuvants are approved for human use [for example, AS01/monophosphoryl lipid, M59/squalene and CpG], so it makes most sense to focus on those in the immediate future. Co-developing a new antigen and a new adjuvant would be more complicated, perhaps too much so in the relevant time frame. The Novavax S protein is combined with the Matrix M adjuvant, which the company has experience with in humans, it seems ${ }^{5}$.

\section{How likely do you think it is that SARS-CoV-2 vaccines will elicit serious side effects, such as ADE and vaccine-associated enhanced respiratory disease?}

K.B.: ADE was found to be the cause of deaths and serious illness of children enrolled in a vaccine trial against respiratory syncytial virus in the 1960 s. In one trial, several children who received the vaccine developed a serious illness when infected with the natural virus. However, we have no evidence so far of ADE occurring in human patients enrolled in SARS-CoV-2 vaccines trials. $\mathrm{T}_{\mathrm{H}} 2$ immunopathology is implicated in $\mathrm{ADE}$, but there is no evidence from studies of T-cell responses in infected individuals for any $\mathrm{T}_{\mathrm{H}} 2$-biased responses. It is hypothesized that faulty $\mathrm{T}$-cell responses trigger allergic inflammation and that low-potency antibodies form immune complexes, activating the complement system and potentially damaging the airways. To explore whether any SARS-CoV-2 vaccine is associated with ADE, NHP studies are used to evaluate safety before vaccines are dosed in human studies and mitigate the potential risks of $\mathrm{ADE}$.

ADE and vaccine-associated enhanced respiratory disease are risks that must be considered as possible but unlikely events. Nevertheless, these risks will need to be monitored carefully throughout the development of the vaccines.
Everyone in the United Kingdom has a National Health Service number and almost everyone has electronic health records, and so the MHRA is able to review real-world data to support postauthorization phase 4 pharmacovigilance (safety) studies to evaluate vaccine safety in real-world populations, as well as other proactive enhanced evaluation of safety through well-established processes within the agency. Longer term safety will also be part of the ongoing phase 3 vaccine trials.

G.A.: I am unconvinced that vaccines will drive ADE. Limited data exist to suggest that SARS-CoV-2 can infect phagocytic cells following antibody opsonization; however, antibodies can certainly trigger inflammation that may contribute to disease pathology. However, emerging data from super-susceptible hamsters suggest that, at low monoclonal antibody titers, low levels of weight loss may occur. However, at higher antibody titers, no pathological effects are observed. Yet there are no data from monkeys or humans to suggest that pre-existing antibodies aggravate or enhance disease. As the rates of infection continue to accrue in some part of the world, studies in previously seropositive individuals or at durability time points in vaccine studies may provide insights on whether ADE may occur with waning antibody titers. But I believe the ADE effect observed with [vaccinia virus Ankara] MVA-CoV-1 that was published in 2019 raises a critical issue - that we must be vigilant, as different vaccines can induce different 'flavors' of antibodies, which may drive inflammation. These data suggest to me that $\mathrm{Fc}$ profiling, beyond ADE assays, must be embedded in all the vaccine development programs so we can learn rapidly and in real time to address any potential vaccine liabilities.

J.M.: Answers will only be possible once enough vaccine recipients have become 
infected in phase 3 trials. There are, and will remain, concerns about these issues until hard data emerge. So far, the animal studies, particularly in macaques, have looked encouraging from the safety perspective. But, in the end, only human data from a substantial number of cases will alleviate nagging worries about postinfection adverse events, particularly for vaccines that induce neutralizing antibodies relatively poorly.

R.R.: The trials need to monitor ADE. There has been a lot of discussion about it, and there is no reliable in vitro or animal model. So the only way to study ADE is to look out for it in clinical trials. Personally, I do not believe this is a risk for the vaccines that induce good titer of neutralizing antibodies, but we need to monitor it and have a definitive answer for it.

\section{How have vaccine developers gone about accelerating clinical development?}

G.A.: It is important to convey that no corners are being cut. In fact, the large developers have now signed a pledge guaranteeing that safety will be a top priority. The major change here is that the pipeline is simply being accelerated and the sequence of activities has simply changed. The antiquated days of having multiple preclinical studies performed before phase 1 , then phase 2 , then phase 3 sequentially is gone. Efficiencies have been created in the system that still enable iterative learning. Combining phases with go/no-go decisions cuts months of vaccine trials. In addition, preclinical studies run in parallel offer a unique opportunity to blend in endpoint readouts as the trials progress. However, it is critical to note that this is all only possible because a large body of data already exists for many established vaccine platforms. Thus, this is not a total surprise. However, for the new products, the go/no-go decisions must be stricter.

It is also important to acknowledge the massive financial risks that pharmaceutical companies have undertaken to make millions or billions of doses of their vaccines before vaccine approvals. Massive investments from the NIH [US National Institutes of Health], CEPI [Coalition for Epidemic Preparedness Innovations], Gates Foundation and other national funding agencies have helped support the commitment that vaccine developers have made. This commitment ensures that any vaccine that looks promising can move forward.

Thus it is critical to remember that scrutiny has not gone away - the criteria have not relaxed. It is that data have to be collected faster and funding decisions have to be taken 'at risk'. Adaptive trials have also been implemented, enabling the integration of additional demographic populations, more/less vaccine boosting, and changes in regimen. Together, these changes are likely to reshape the way in which we develop vaccines, once taking decades.

K.B.: Assessment of safety remains the primary and fundamental goal of all vaccine trials, and then assessment of efficacy. The design of the SARS-CoV-2 vaccine studies are no different to any other vaccine trials, although the later phases of SARS-CoV-2 clinical development are currently being compressed and run in parallel. The reason for this parallel clinical development in the SARS-CoV-2 vaccine trials versus sequential development in traditional vaccine trials is that funding has been unlocked to accelerate clinical development to end the pandemic through vaccination as quickly as possible.

For example, unlike in traditional clinical development, SARS-CoV-2 vaccine phase 2 and phase 3 trials have been funded to start recruiting as soon as the initial phase 1 safety has been established. When the Oxford team published ${ }^{8}$ their phase $1 / 2$ neutralizing antibody results with ChAdOx1, they had by then already vaccinated 8,000 people in their large efficacy phase $2 / 3$ trials, which was only possible due to unprecedented levels of international organization and cooperation. We need to try to continue this fabulous level of cooperation and speed in future vaccine development.

L.C.: No corners are being cut in the clinical development of coronavirus vaccines. Phase 1 and 2 trials are of normal size and numbers, and all safety and immune assessments are evaluated by study teams as well as the FDA before granting permission for the next stage of human testing. In general, the time from phase 2 to phase 3 of a trial is often a year. Here it's 7-14 days - all because the logistics and operational support of a large-scale phase 3 trial are in place at the time of the phase 2 discussion.

The primary endpoint for all the phase 3 vaccine trials is amelioration of COVID-19 disease. One can think of this as defining COVID-19 into severe and non-severe disease. Most vaccines have shown greater benefit in reducing the disease burden of persons with severe disease more effectively than mild disease. Thus, one goal of the phase 3 trial is to enroll persons at high risk of severe disease. The definition of disease severity is complex and is the crux of [vaccine strategy in] meeting this disease.

Who are the optimal target populations for phase 3 trials, and how important are genetic background, age and other factors in the general population in determining vaccine response?

G.A.: Age, genetics and sex all tune

vaccine-induced immunity. Emerging data clearly demonstrate significant differences across the sexes with respect to disease pathology, with enhanced disease in age-matched men. Conversely, women tend to make stronger humoral immune responses following vaccination. Yet whether the correlates will differ as well following vaccination is unclear. Phase 3 trials in adults have been designed powered to define the influence of sex as a critical biological risk factor. Moreover, phase 3 trials have been designed to also test for protection in the elderly, a critical vulnerable population. Unfortunately, pregnant woman and infants are always last, together with immunocompromised populations and those [with underlying conditions] on medications, to be integrated into vaccine studies and thus will remain vulnerable for longer periods of time. Protection of this population will be key until safety can be ascertained in these immunologically distinct populations.

But we are in a unique situation with the SARS-CoV-2 vaccine development pipeline, where many vaccine strategies are being tested simultaneously, and some platforms may induce better immunogenicity/durability in pregnant women, other may work more robustly in the elderly, and another may be highly potent in infants. This flexibility and range of options is unparalleled and may help us understand platform advantages for SARS-CoV-2 and beyond.

K.B.: The MHRA have specified that phase 3 trials should include volunteers covering all age groups and demographics but that these trials should be enriched with the target populations at the greatest risk of severe illness and mortality from COVID19. Almost all vaccine developers are not choosing to evaluate their SARS-CoV-2 vaccines in children less than 18 years old in their current large efficacy trials.

In the United Kingdom, the groups that have been prioritized for vaccination, if and when a vaccine is available, include frontline health and social care workers (who are at increased personal risk of exposure to infection and of transmitting that infection to patients in health and social care settings) and those people at increased risk of serious disease and death. This includes all adults over the age of 50 (with the risk increasing with age), 18- to 50-year-olds with underlying comorbidities including chronic heart disease, chronic kidney disease, chronic pulmonary disease, malignancy, diabetes, obesity and dementia, all shielded people (who are at greatest risk of severe illness) and certain Black and minority ethnic groups. (The reasons behind the apparently disproportionate effects 
on certain ethnic minorities are complex, poorly understood and undergoing further investigation.)

The impact of genetics in determining response to vaccines is not yet known, and I hope the data may come out of the numerous trials worldwide. It is well known that aging negatively impacts the production of $\mathrm{B}$ and $\mathrm{T}$ cells and dampens the function of mature lymphocytes in secondary lymphoid tissues. As a result, the elderly do not typically respond to immune challenge as robustly as the young, so they need vaccines with adjuvants or other components to rejuvenate the immune system and evoke a strong immune response. Pre-existing exposure may also affect the vaccine response. Safety is the primary driver for vaccination of children. I expect there will ultimately be different vaccines for different population cohorts.

L.C.: Epidemiological data indicate the target populations must be older adults (over 65) and those with comorbidities, such as heart or lung disease, hypertension, diabetes and obesity. Black and Latinx populations are associated with severe infection, especially in the under-65-year-old population. This likely relates to high rates of exposure due to their occupations and living density.

Many protein-based vaccine platforms have reduced immunogenicity in older adults. Given that older adults have increased susceptibility clinically to both SARS-CoV-1 and SARS-CoV-2, as well as increased severity when facing SARS-CoV-2 in human and experimental challenge, whether disease enhancement with vaccines is seen in older adults is at present unknown. Early data from the RNA phase 1/2 [vaccine trial] indicate good responses to this vaccine in those over 65 years of age. We await further data to see whether this is associated with good efficacy.

J.M.: Too little information has been disclosed on these aspects so far for me (or anyone?) to know what to say here. And that's far from ideal as the questions are important ones. In the end, these vaccines do need testing in higher risk populations, including but not limited to older people. Vaccine immunogenicity is known to diminish with age, in general terms, and it's hard to see why that would be any different for COVID-19 vaccines. Recent data, published by Pfizer/BioNTech and in a Moderna, indicate that antibody responses are a few-fold weaker in people over 55 than in younger volunteers, but not to an extent that's a serious concern.

R.R.: One of the limitations of the trials may be whether we obtain data from a population that is genetically sufficiently different. However, trials can only be performed in those areas where there are enough cases. Hopefully, we will be able to establish correlates of protection that can be useful to extend the information also to other populations and to different ages, including children.

\section{In the context of a pandemic, what standards should regulatory agencies use to assess a COVID-19 vaccine?}

G.A.: Both efficacy and immunogenicity endpoints should be synchronized across all vaccine platforms. For many platforms that will be tested under the COVID-19 Prevention trials network, standardized tests will be used to quantify disease severity and vaccine immunogenicity. Additionally, efficacy will be evaluated by centralized statisticians with tremendous experience in vaccine efficacy analysis.

However, for most platforms, minimized disease severity will be used as a primary end point. Unquestionably, reducing severity will profoundly improve our capacity to manage this infection at a global level; however, multiple endpoints should be evaluated, including infection (seroconversion), viral RNA levels, and even secondary pathology such as cardiac complications. Unfortunately, the trials are not powered to encompass all these end points; however, post hoc analyses of these additional end points may provide additional insights across the platforms that may be vital for the downselection and evaluation of vaccines able to drive the most robust disease-modulating effects.

K.B.: The standards for a SARS-CoV-2 vaccines are no different from any other vaccine: this is outlined in the European Union guidelines. The time to regulatory approval may be shortened, but standards will not be compromised.

Seroconversion alone is not sufficient where the correlate of protection has not been established by prior work, such as from other COVID-19 vaccines. Once this has been established with a degree of certainty, which means having convincing efficacy and safety data from several vaccines, then approaches such as immune-bridging may be justified, alongside a safety database of subjects enriched in the target populations to be vaccinated. In the United Kingdom, the target groups for COVID-19 vaccination is specified by the Joint Committee on Vaccination and Immunisation (JCVI) and includes the elderly, adults with comorbidities, ethnic minorities and frontline workers, representing over $50 \%$ of the UK adult population.

The primary objective is typically to evaluate the rate of virologically confirmed (by PCR) COVID-19 symptomatic disease cases, regardless of severity, with the primary end point being the first COVID-19 disease episode occurring more than 14 days after the first dose. Other definitions for the primary endpoint may also be acceptable. Disease severity, including asymptomatic disease, is a key secondary endpoint. T-cell responses, while gaining interest, are not currently critical for approval as there are currently insufficient data to explain the implications of cellular responses, although emerging data classifying patients by TCR sequence suggest they may turn out to be as important as measuring antibody levels. J.M.: If the COVID-19 vaccines are going to be widely used (and they will be of limited value if they are not), then the public must have strong confidence that they are both safe and effective. That means the approval process has to be both thorough and transparent and, above all, free of political interference. Multiple opinion polls now clearly show that vaccine uptake by the public would diminish significantly if there were a repeat of the hydroxychloroquine EUA [Emergency Use Authorization], in which election politics dominated a transparent and trusted scientific process. A vaccine that's not trusted is a vaccine that's not used. And a vaccine that's not used is a vaccine that's not effective.

R.R.: I believe it is imperative to demonstrate efficacy for severe disease and also for infection of the upper respiratory tract. It will be very important to use this information to establish correlates of protection. Indeed, it will not be possible to perform efficacy trials for all vaccines in development, and correlates of protection will be the only road for licensing several vaccines.

\section{Should regulators gather cross-trial data from all vaccine trials to make decisions on best comparative evidence, as has been proposed ${ }^{13}$ ?}

G.A.: Cross-comparative analysis will be very difficult, and the urgency for a SARS-CoV-2 vaccine should not 'break the system'. Cross-comparative data are not as critical as the ultimate development of a safe and effective vaccine. For each vaccine a Drug Safety Monitoring Board (DSMB) will be assembled. We have to have confidence in these experts that data will be reviewed thoughtfully, objectively and with extreme rigor. These confidential, closed meetings, along with the FDA and other regulatory bodies, will provide the evaluation of the vaccine results that are required to move a promising product safely into humans. In the meantime, public disclosures in the form of presentations, 
Table 2 | Peer-reviewed papers describing clinical trial results from SARS-CoV-2 vaccines in late-stage development

\begin{tabular}{|c|c|c|c|}
\hline Sponsor(s) & Vaccine type & $\begin{array}{l}\text { Clinical } \\
\text { stage }\end{array}$ & Peer-reviewed papers \\
\hline $\begin{array}{l}\text { Wuhan Institute of } \\
\text { Biological Products, } \\
\text { Sinopharm }\end{array}$ & Inactivated virus & Phase 3 & $\begin{array}{l}\text { Ref. }{ }^{14} \text { : "Effect of an inactivated vaccine against SARS-CoV-2 } \\
\text { on safety and immunogenicity outcomes. Interim analysis of } 2 \\
\text { randomized clinical trials" }\end{array}$ \\
\hline $\begin{array}{l}\text { Moderna, NIAID, } \\
\text { Lonza, Catalent, } \\
\text { Rovi, Medidata, Bioqual }\end{array}$ & $\begin{array}{l}\text { RNA-based vaccine (nucleoside-modified mRNA) } \\
\text { encoding codon-modified S protein encapsulated } \\
\text { in ionizable LNPs, containing heptadecan-9-yl } \\
\text { 8-((2-hydroxyethyl)(6-oxo-6-(undecyloxy) } \\
\text { hexyl)amino)octanoate (Lipid 8), 1,2-distearoyl } \\
\text { phosphatidylcholine, cholesterol and polyethylene } \\
\text { glycol lipid in 50:10:38.5:1.5 ratio }\end{array}$ & Phase 3 & $\begin{array}{l}\text { Ref. }{ }^{15} \text { : "An mRNA vaccine against SARS-CoV-2 - preliminary } \\
\text { report" }\end{array}$ \\
\hline $\begin{array}{l}\text { CanSino Biologics, } \\
\text { Beijing Institute of } \\
\text { Biotechnology, National } \\
\text { Research Council of } \\
\text { Canada }\end{array}$ & $\begin{array}{l}\text { Non-replicating viral vector (Ad5 vector encoding S } \\
\text { protein) }\end{array}$ & Phase 2 & $\begin{array}{l}\text { Ref. } 6 \text { : "Safety, tolerability, and immunogenicity of a } \\
\text { recombinant adenovirus type-5 vectored COVID- } 19 \text { vaccine: a } \\
\text { dose-escalation, open-label, non-randomized, first-in-human } \\
\text { trial"; ref. ": "Immunogenicity and safety of a recombinant } \\
\text { adenovirus type-5-vectored COVID-19 vaccine in healthy } \\
\text { adults aged } 18 \text { years or older: a randomized, double-blind, } \\
\text { placebo-controlled, phase } 2 \text { trial" }\end{array}$ \\
\hline Novavax & $\begin{array}{l}\text { Recombinant subunit vaccine with full-length } \\
\text { prefusion spike protein in a nanoparticle, Matrix M } \\
\text { adjuvant }\end{array}$ & Phase $1 / 2$ & $\begin{array}{l}\text { Ref. 5: "Phase 1-2 trial of a SARS-CoV-2 recombinant spike } \\
\text { protein nanoparticle vaccine" }\end{array}$ \\
\hline $\begin{array}{l}\text { Gamaleya Research } \\
\text { Institute of } \\
\text { Epidemiology and } \\
\text { Microbiology }\end{array}$ & $\begin{array}{l}\text { Two-component vaccine: recombinant } \mathrm{Ad} 26 \text { and } \\
\text { Ad5, both carrying spike protein }\end{array}$ & Phase $1 / 2$ & $\begin{array}{l}\text { Ref. }{ }^{16:} \text { "Safety and immunogenicity of an rAd26 and rAd5 } \\
\text { vector-based heterologous prime-boost COVID- } 19 \text { vaccine } \\
\text { in two formulations: two open, non-randomised phase } 1 / 2 \\
\text { studies from Russia" }\end{array}$ \\
\hline $\begin{array}{l}\text { University of Oxford, } \\
\text { Oxford Biomedica, } \\
\text { AstraZeneca }\end{array}$ & $\begin{array}{l}\text { Non-replicating viral vector (chimpanzee adenoviral } \\
\text { vector encoding S protein; AZD 1222; formerly } \\
\text { ChAdOx1) }\end{array}$ & Phase $1 / 2$ & $\begin{array}{l}\text { Ref. } 8 \text { : "Safety and immunogenicity of the ChAdOx1 nCoV-19 } \\
\text { vaccine against SARS-CoV-2: a preliminary report of a phase } \\
\text { 1/2, single-blind, randomised controlled trial" }\end{array}$ \\
\hline
\end{tabular}

public forums and published manuscripts will be important for the public's ability to evaluate the data.

K.B.: Head-to-head trials would only be possible if there were a vaccine already licensed. And even then, early vaccines would likely have conditional approval so may not be considered appropriate comparators. The European Medicines Agency's guidelines suggest that if one vaccine is already approved for prevention of the disease then it may be difficult to identify a study population that still has a sufficient incidence of disease to allow for reliable estimates of efficacy to be made.

Typically regulators, including the UK's MHRA, do not compare data from different vaccines unless there is a specific reason, such as immuno-bridging comparisons. The main consideration for regulators is to assess whether any vaccine meets the requirements for a license; it is not the role of the regulators to choose one vaccine over another.

Common oversight is possible, however. For example, the AstraZeneca/Oxford University vaccine effort has a single DSMB for their UK, Brazil and South African clinical trials, but I'm not aware of DSMBs being common across different companies or products.

The UK VTF, however, does want to compare the data generated in different vaccine trials so that the JCVI can make more informed recommendations on vaccine selection and advise the UK government on strategy. So we are investing in building standardized, accredited assays, including quantitative high-throughput S-protein ELISA assays, live viral neutralization assays and T-cell assays. We are fortunate to be able to work with Public Health England Porton Down to build on their viral neutralization assay capability, which is already being used to support clinical trials. Once accredited, these assays will be available to any vaccine developer or country to use.

J.D.: I support the idea of a cross-trial DSMB. In order for people to take the vaccine, they need to trust information they have received. The best way to create trust is through a coordinated, coherent and transparent public conversation. It will be critical to accurately state the benefits and risks of the vaccine because even a single severe side effect will be covered and shared extensively on social media. Put another way, the 'credibility' of the vaccine cannot depend on it being a perfect product without any chance of side effects.

J.M.: They should, but will they? Companies who may fear/know their products will fare poorly in a head-to-head matchup may well find reasons to not take part.

What are the pros and cons of each vaccine modality in terms of large-scale manufacture?

K.B.: Scale-up to large-scale manufacturing of all COVID-19 vaccine modalities is a challenge, given the speed of all the programs. Manufacture is better understood for some vaccine modalities than others. For example, viral-vector vaccines are very similar to gene-therapy technology, a modality that has more history than, for example, mRNA vaccines. The United Kingdom has plenty of experience manufacturing gene therapy vectors for therapeutic use. RNA vaccines are less well known in general, and to date no RNA vaccines have yet been registered for commercial use. An additional manufacturing complexity of mRNA vaccines is the LNP technology required to 
stabilize them, but ultimately this should not be an obstacle.

There are differences in the productivity of different modalities, and each modality has different dose requirements as a vaccine. Protein and adeno vaccine modalities can generally produce millions of doses from 2,000-liter to 4,000-liter scale, but the productivity/yield depends largely on the individual vaccine, the process parameters, and the skills and capability of the people developing and making them.

The mRNA and self-amplifying RNA vaccines are very active in very small quantities so many millions of doses can be produced in relatively small volumes. However, these vaccines need to be formulated in LNPs, which is a challenging process that currently reduces the overall productivity. The human capability is extremely important, particularly in the rapid scale-up processes underway today, which is why the UK VTF has invested funds to meet the specialist skills demand for vaccine manufacture in the United Kingdom. N.J.: RNA is fast to clinic, but not of high productivity in manufacturing to date. The issue is the maturity of the platform and hence the lack of established large-scale manufacturing. This is an obstacle that can be overcome with time, but meanwhile scale-up will be expensive. Cost of goods is also high as the batch sizes are small and raw materials are not available in large quantities with the sufficient purity. Finally, RNA products may require ultra-low cold chain to maintain stability of the RNA and/or LNP delivery vehicle.

DNA is fast to clinic, but requires rather high doses, making manufacturing a challenge. Also, the DNA vaccine requires manufacture of an electroporation device for delivery of the product, which adds significant cost and further limits supply availability. The production process is mature, so many CMOs [contract manufacturing organizations] exist that can make large quantities.

Live viral vectors are proven technology, are generally scalable and have good yields. The process is fairly simple and the downstream purification is minimal, allowing higher yields/recovery. One drawback is that these vectors generally require cold chain $\left(-20\right.$ to $\left.-80{ }^{\circ} \mathrm{C}\right)$ to preserve the viral activity.

Manufacturing for subunit protein vaccines is well established, and for $S$ protein in the trimeric form the proteins are very stable, even at elevated temperatures. They require an adjuvant to boost immune response, and in most cases capacity of adjuvant is more limiting than our capacity to make the recombinant protein. The products are highly purified, which reduces yield of production to some extent, but the processes are still able to exceed 1,000 doses per liter (up to 5,000 doses per liter).

R.R.: Manufacturing of hundreds of millions or billions of doses will be a big challenge for all vaccines, especially for the new ones, such as those based on nucleic acids and viral vectors, that have never been manufactured in large quantities. Fortunately, an unprecedented amount of money is being invested at risk to accelerate the scale-up of these platforms. However, we know that industrialization of vaccine manufacturing takes time, and it is not done until it is done. So, while we all hope that we will be successful, we need to continue to be prepared for surprises.

\section{What are the challenges in terms of vaccine filled and finished product, cold chain, storage/stability, distribution and administration for the various vaccines?} K.B.: While there are challenges in the fill-finish process itself, there is a global shortage of fill-finish capacity and of some components to support all the vaccine development efforts at a global scale right now. Anticipating this, we have acquired several million doses of fill-finish capacity so we can receive bulk drug substance for fill-finish in the United Kingdom.

Because vaccines are being developed in real time, there is no historical stability data, so stability data are being generated for the regulator as the development process proceeds. It is not possible to accelerate stability testing to extrapolate to a potentially longer shelf life, so in the short term, while a longer stability is verified, it will be a big challenge to match delivery of vaccines with the capacity to administer. For the vaccines stable at $2-8{ }^{\circ} \mathrm{C}$, such as adeno and adjuvanted protein vaccines, this will be somewhat easier; however, those that require very cold storage will need a very well thought-through deployment strategy. J.M.: RNA vaccines have to tackle the issue of thermal fragility; it's also quite hard to imagine how a DNA-vaccine electroporation device could be used on anything more than niche applications. It would be a tough sell even in well-equipped hospitals, let alone in more challenging low-resource environments. For it to be worth going there, the DNA vaccine would have to be far superior to its competitors. It's hard to see that happening. All things being equal, and they may not be, the vaccine that's easiest to distribute and use may be the vaccine that's most useful on a global scale.

R.R.: A challenge underestimated by many is the capacity to fill billions of doses of vaccines. In any case, vaccines in large quantities are likely to become available only in the second half of 2021, and until then, it will be important to prioritize the people at risk who will need the vaccines first.

\section{How should we think about the ques- tion of achieving herd immunity through vaccination?}

G.A.: Social network analyses could guide the strategic generation of herd immunity. It is simple nowadays to define the individuals who sit at the center of most social networks. Using these strategies, individuals on the front lines (for example, teachers, drivers, hospital works, grocery store workers, hairdressers, dentists) will likely be the hubs of most networks and should get a top-tier status for vaccination. Additionally, once a vaccine is approved, vulnerable populations at the center of networks (for example, nursing homes) should gain priority. This would be a sociological experiment, but could help make deployment equitable and guide rational deployment protocols similar to ring vaccine efficacy trials. K.B.: Clinically vulnerable populations should be prioritized for vaccination, but vaccinating these populations alone is unlikely to achieve population herd immunity to control the pandemic worldwide. Herd immunity is likely to be derived from vaccination and continuing infections. I think it is unlikely that we will reach a level of herd immunity that is required, owing to the large number of asymptomatic patients where the induction of lasting immunity (including T cells) is unknown.

If a vaccine were shown to be safe and provide durable protective immunity, then it is possible that the risk/benefit balance will allow all adults to be vaccinated, rather than just those who are clinically vulnerable. In that case, herd immunity could be achieved. We need to vaccinate the majority of the population to keep [the reproduction number] $R$ below 1 .

However, there are several issues to consider regarding herd immunity, including longevity of response, type of response required for herd immunity, and kinetics and pattern of herd immunity (epidemiology), which are complicated and not currently well understood.

J.M.: To achieve herd immunity requires vaccinating a major proportion of the population (maybe as high as $70 \%$ in some models) with a product that's substantially protective. Logically, one would try to first protect those most at risk of infection and severe disease, but while we know some of the parameters influencing disease, we have little idea of the risk factors involved 


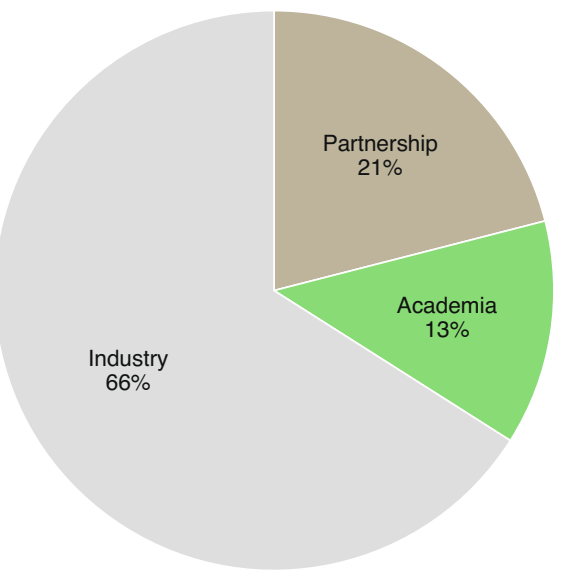

Vaccine developers. Adapted with permission from ref. ${ }^{17}$, Springer Nature.

in acquiring and spreading the virus. I hope there actually is a plan, but I have read only proposals and opinions so far.

\section{What are the best mechanisms to achieve equity in vaccinat- ing under-resourced and high-risk communities?}

K.B.: The United Kingdom is committed to ensuring that people around the world have fair access to a safe and effective COVID-19 vaccine. It is highly unlikely that any one country will develop the only successful vaccine and be able to manufacture all its component parts at sufficient speed and scale for global use.

This is not a time for nationalist politics. China, the United States, Europe and the United Kingdom need to work together. We need to collaborate internationally to pool risks and costs, address barriers to access, and scale up manufacturing capacity internationally to produce sufficient doses of any COVID-19 vaccine.

The United Kingdom is therefore a strong supporter of COVAX, a multilateral initiative to support discovery, manufacture and fair distribution of COVID-19 vaccines. We are working to set this up with a broad alliance of some 180 nations and international organizations including GAVI (the Global Alliance for Vaccines and Immunization, a global health partnership of public- and private-sector organizations that delivers childhood vaccinations to almost half of the world's children), CEPI (Coalition for Epidemic Preparedness Innovations) and the WHO (World Health Organization) to ensure equitable access of COVID-19 vaccines to low-income countries and high-risk as well as middle- and high-income countries.
The United Kingdom supports this global approach for equitable access to vaccines and is one of the largest country funders of both CEPI and the GAVI alliance.

As in all global health matters, there are multiple stakeholders with many competing interests trying to balance the challenge of national and global interests. The COVID19 pandemic has affected all countries, both rich and poor, and unusually in this first phase of the pandemic, high-income countries have been more affected than low-income countries, which changes the balance of national interests, global interests, domestic funding and overseas development-aid-style funding.

So the best mechanism to help end the global COVID-19 pandemic is for the high-income countries to come together to provide funding and support to ensure that a COVID-19 vaccine should be made available as rapidly and widely as possible - to high-, medium- and low-income countries - for the health of the global economy and global population. It is highly unlikely that any one country will develop the only successful vaccine and be able to manufacture all its component parts at sufficient speed and scale for global use.

J.D.: Vaccine distribution will almost certainly become a political flashpoint. I am especially worried that vaccine distribution will be turned into 'us versus them', both across countries (that is, vaccine nationalism) as well as within a country (that is, vaccine partisanship). The United States is already a partisan powder keg, and a contentious election is around the corner. As a result, there is a real possibility that under-resourced communities will not receive enough vaccines, even if their communities are high-risk. This will need to be combatted actively, and to that end, I favor a weighted lottery system, wherein people who are more likely to be exposed are more likely to receive the vaccine N.J.: Making vaccines available to those who need them most is the fastest, as well as the fairest, way to bring the pandemic to an end. We anticipate that initial demand for COVID-19 vaccines is going to vastly outstrip supply; every country on the planet will need access to vaccines at the same time, to protect their most vulnerable populations. But that is already leading to a race between countries to secure vaccine supply for their own population. This approach will push countries to the back of the queue if they can't afford to make deals with manufacturers and leave many of those who are most vulnerable to the virus unprotected.
To address this challenge, CEPI, alongside GAVI and the WHO, launched COVAX as part of the Access to COVID-19 Tools (ACT) Accelerator on 4 May 2020, with the aim of ending the acute phase of the pandemic by the end of 2021. COVAX is a global initiative that aims to provide an end-to-end solution to the challenge of vaccine development, manufacture and supply in this pandemic, bringing together the skills, expertise and resources of the public, private and philanthropic sectors on a global scale.

COVAX is the only solution that will deliver fair, equitable and necessary access to vaccines for every country that participates. It aims to produce two billion doses of vaccine and distribute them according to an equitable allocation formula in 2021. Countries of all income levels can participate in the COVAX Facility, a financing mechanism established by the COVAX partners. All participating countries, of every income level, will get doses for at least $20 \%$ of their populations. That covers those most at risk: health workers, older people, and adults living with health conditions.

J.M.: Build data-driven trust that the vaccine is both safe and effective, which requires a thorough and transparent approval process. And then provide the financial and logistical resources needed to roll out the vaccine everywhere it's most needed. In the face of a global pandemic, 'vaccine nationalism' is an abhorrent concept, but it will be hard to counter.

How do you compare the vaccine choices made by Operation Warp Speed and the UK Vaccine Taskforce?

K.B.: I have regular conversations with Moncef Slaoui, who runs Operation Warp Speed (OWS), and the United States is astonishingly fortunate to have such a remarkable and thoughtful expert in vaccines, with deep experience and compassion, to help develop and deliver COVID-19 vaccines at speed. The choices that OWS have made overlap in part with those made by the UK VTF, and I hope that several vaccines are successful this and next year.

An important positive outcome of the COVID-19 pandemic that I have observed is the open, regular and transparent interactions between governments, companies, regulators and academic institutions, all of which have served not only to bring together the best brains in the world with immediate funding but also to accelerate the development of COVID-19 vaccines that can both help stop this pandemic and restore global 
economic health and growth. We must ensure this level of collaboration and global cooperation continues.

J.D.: I do not have concerns with the VTF. I am concerned about politics influencing OWS. The number of lies told by the US administration is shocking, and a growing number of statements (for example, overselling the benefits of plasma) and actions (for example, changing guidelines so asymptomatic patients do not necessarily need to be tested) by scientific institutions clearly demonstrates that the executive branch is choosing politics over truth. I am therefore concerned that OWS will be pressured to make politically driven decisions. That said, I am hopeful that the leading scientists helping with OWS will resist this pressure.

J.M.: Both countries seem to have adopted the 'get there the firstest with the mostest' philosophy. In other words, something that can be made 'tomorrow' was emphasized over what could be produced 'next week'. That's peachy if it all works, but there's always a concern about cutting corners when there's a need for speed.

An additional dynamic is that the various companies are competing with each other, which is understandable, given the financial stakes and the prestige involved (the latter particularly for the academic collaborators). Again, that could lead to corner-cutting, particularly by smaller companies that are 'one-trick ponies'. Cooperation would be much more constructive and, in some scenarios, might be necessary (for example, for combination vaccines, a 'plan B' option). R.R.: From what I can see from the media, the choices made by OWS and the VTF reflect the uncertainty of the field. In the absence of sufficient data about safety, efficacy, large-scale manufacturing, and the stability of the different vaccines in development, they tried to book vaccines from several different platforms, hoping that some of them will be the right choice - a wise strategy given the limited information we have.

What are your closing thoughts and best-case scenario for public health control of SARS-CoV-2?

G.A.: We need to understand the nature of reinfection. Natural correlates of exposure could significantly change the game and give us insights to drive vaccine development and, more importantly, to prioritize particular vaccines for those that are most vulnerable to disease. In the meantime, social distancing will be a reality for the foreseeable future. This should be emphasized. We will not be out of masks for a long while. The economy continues to suffer, and we need creative solutions to help our population and world heal. This will not be the last pathogen, and if we learn only one lesson, let us continue to make diagnostics and vaccines a priority, so we can stop emerging infections in their tracks and prevent a pandemic from paralyzing our globe again.

K.B.: The best-case scenario for public health control of SARS-CoV-2 is that we find a cheap, stable sterilizing vaccine. Assuming that doesn't happen (immediately), the next best case is we have multiple vaccines for different at-risk populations, which are disease modifying, lessen the severity of the infection, and (together with effective therapeutics) reduce mortality from COVID-19. I am optimistic this will happen.

However, we have to recognize that we are likely to face future evolution of the biology of the SARS-CoV-2 virus and also of future viruses. So this means we need to improve our global public health preparedness, where vaccines are just one piece of the pie.

J.D.: My best-case scenario for public health control of SARS-CoV-2 is not scientific. Instead, it is social: a return to truth-based conversations. Politicians must acknowledge and appreciate the value of objective scientific evidence. Until we return to these norms, SARS-CoV-2 will remain a social and political issue and, as a result, scientific and public health solutions to COVID-19 will be encumbered.

In the United States, scientific and medical professionals must continue to highlight the need for everyone to wear masks. I am in awe of our frontline medical workers, many of whom have spent time away from family, gotten sick, or died. Frontline medical professionals are willing to make the ultimate sacrifice for us, and yet many of us will not make the small sacrifice of wearing a mask for them.

J.M.: My best-case scenario is that several vaccines are proven to be safe and effective and can be very widely used throughout 2021. Combined with better therapies, and also with more traditional methods (masks, social distancing, lockdowns), vaccines could end the pandemic next year.

Company executives at vaccine manufacturers (and politicians) really need to pay much more attention to the need to boost public confidence in their products. Vaccine hesitancy is a real thing, and it's becoming worse during the ultrapoliticized COVID-19 pandemic. Creating a vaccine that is effective but not widely used because it's not trusted would be a very expensive Pyrrhic victory .... In other words, some companies may need to rein in their ambitions to be first, as they will need the support of the vaccine science and public health communities. If the latter believe or suspect that shortcuts have been taken with safety, that support may not be a given.

\section{Interviewed by Laura DeFrancesco}

Published online: 28 September 2020 https://doi.org/10.1038/s41587-020-0697-7

References

1. Moore, J. P. \& Klasse, P. J. J. Virol. 94, e01083-e20 (2020).

2. Pallesen, J. et al. Proc. Natl Acad. Sci. USA 114, E7348-E7357 (2017)

3. Mercado, N. B. et al. Nature https://doi.org/10.1038/s41586-0202607-z (2020).

4. Corbett, K. S. et al. N. Engl. J. Med. https://doi.org/10.1056/ NEJMoa2024671 (2020).

5. Keech, C. et al. N. Engl. J. Med. https://doi.org/10.1056/ NEJMoa2026920 (2020).

6. Zhu, F. C. et al. Lancet 395, 1845-1854 (2020).

7. Zhu, F. C. et al. Lancet 396, 479-488 (2020).

8. Folegatti, P. M. et al. Lancet 396, 467-478 (2020).

9. Paunovska, K. et al. Sci. Adv. 6, eaba5672 (2020).

10. Lokugamage, M. P. et al. Adv. Mater. 32, el904905 (2019).

11. Patel, S. et al. Nano Lett. 17, 5711-5718 (2017).

12. Jackson, N. A. C. et al. Vaccines (Basel) 5, 11 (2020).

13. Corey, L., Mascola, J. R., Fauci, A. S. \& Collins, F. S. Science 368, 948-950 (2020).

14. Xia, S. et al. J. Am. Med. Assoc. https://doi.org/10.1001/ jama.2020.15543 (2020).

15. Jackson, L. A. et al. N. Engl. J. Med. https://doi.org/10.1056/ NEJMoa2022483 (2020).

16. Logunov, D. Y. et al. Lancet https://doi.org/10.1016/S01406736(20)31866-3 (2020).

17. Shin, M. D. et al. Nat. Nanotechnol. 15, 646-655 (2020). 